\title{
O ENSINO DE GRADUAÇÃO NA ESCOLA DE ENFERMAGEM DE RIBEIRÃO PRETO DA UNIVERSIDADE DE SÃO PAULO - SUAS PROSPECTIVAS
}

\author{
Dulce Maria Vendrúscolo de Freitas* \\ Neide Fávero** \\ Maria Cecília Moraes Scatena***
}

As autoras focalizam o ensino de graduação da Escola de Enfermagem de Ribeirão Preto - USP, através de seu marco histórico e conceitual desde sua fundação na década de 50, revelando que, na década de 80 , o estudo da reforma curricular decorreu de um amplo processo de discussão, o que culminou com a proposta de formação do enfermeiro generalista e a implantação do novo currículo em 1989.

$\mathrm{Na}$ versão das autoras, as prospectivas para o ensino da graduação dependem de uma reflexão das escolas sobre o papel das universidades na reorientação dos serviços de saúde, da formação do aluno voltada para a competência técnica, científica, política para atuar em diferentes níveis de assistência a saúde da população. Um novo modelo educacional exigirá a reorientação dos currículos e a inserção das escolas nos serviços de saúde como elemento estratégico de integração do ensino com a prática profissional.

UNITERMOS: ensino de graduação, educação em enfermagem.

\section{INTRODUÇÃO}

A Escola de Enfermagem de Ribeirão Preto da universidade de São Paulo implantou em 1989 um novo Currículo para o curso de graduação. Para tanto foi necessário um amplo processo de discussão sobre a profissão, o setor saúde, a sociedade e a revisão dos objetivos e conteúdos formais de suas disciplinas,

\footnotetext{
* Prof. Dr. junto ao Departamento de Enfermagem Materno-Infantil e Saúde Pública da Escola de Enfermagem de Ribeirão Preto da Universidade de São Paulo.

** Prof. Dr. junto ao Departamento de Enfermagem Geral e Especializada da Escola de Enfermagem de Ribeirão Preto da Universidade de São Paulo.

*** Prof. Dr. junto ao Departamento de Enfermagem Psiquiátrica e Ciências Humanas da Escola de Enfermagem de Ribeirão Preto da Universidade de São Paulo.
} 
buscando a seqüência e a integração das atividades de ensino e aprendizagem. Para melhor entendermos as propostas e alterações que vêm ocorrendo no ensino de graduação desta Escola, necessário se faz uma abordagem retrospectiva de sua história, sua finalidade, seu compromisso com a comunidade e as condições atuais do país no que se refere à saúde da população brasileira, às políticas de saúde, vivenciando-se o processo de municipalização e as condições do mercado de trabalho para os enfermeiros. (Comissão de Reestruturação Curricular da Escola de Enfermagem de Ribeirão Preto - USP, 1993).

\section{MARCO HISTÓRICO E CONCEITUAL DO ENSINO DA ESCOLA DE ENFERMAGEM DE RIBEIRÃO PRETO DA UNIVERSIDADE DE SÃO PAULO}

Fundada em 1951, anexa à Faculdade de Medicina de Ribeirão Preto, iniciou seu curso em 1953, tendo como fundadora e diretora a Profa. Glete de Alcântara. A filosofia e os objetivos da Escola de Enfermagem de Ribeirão Preto - USP na época, refletiam o desejo de se encontrar respostas às necessidades de saúde da comunidade, com o estabelecimento de uma estrutura didático administrativa inovadora, que tinha por objetivos a formação de profissionais para prestação de assistência e direção de unidades de enfermagem em hospitais, centros de saúde, incluindo-se nessas funções o ensino e a supervisão de enfermeiros e auxiliares de enfermagem, com ênfase na administração hospitalar.

Nas décadas de 50 a 60 estava implícito o marco conceitual do ensino da graduação voltado para a comunidade, uma vez que a criação da Faculdade de Medicina e do Hospital das Clínicas exigia a formação e preparo de enfermeiro para a assistência e o treinamento de pessoal para o serviço de enfermagem; a divulgação do trabalho do enfermeiro; o recrutamento de alunos para o curso; preparo de pessoal auxiliar para a área hospitalar e de saúde pública. Como currículo inovador na área da enfermagem incluía a integração dos aspectos preventivos e curativos na saúde, com enfoque na administração de serviços hospitalares e de saúde pública.

Em maio de 1964 a Escola de Enfermagem de Ribeirão Preto da Universidade de São Paulo é desanexada da Faculdade de Medicina de Ribeirão Preto tornando-se estabelecimento de ensino superior, adquirindo sua autonomia didática administrativa, passando os vestibulares para ingresso de alunos a serem efetuados pelo CESCEM em 1969 e posteriormente, na década de 70 pela FUVEST.

A partir de 1967 a Escola de Enfermagem de Ribeirão Preto - USP passou a oferecer o curso de enfermagem geral em 3 anos e mais um ano opcional curso de Enfermagem em Saúde Pública; em 1969 o aluno podia optar no $4^{\circ}$ ano, pela 
formação em Enfermagem Médico Cirúrgica, Enfermagem Psiquiátrica e Enfermagem Pediátrica, situação que se manteve até 1975 com a vigência das Habilitações em Enfermagem Médico Cirúrgica, Enfermagem Saúde Pública e Enfermagem Obstétrica de acordo com o parecer 163/72 do CFE. Em 1976 amplia-se o número de vagas de ingressantes, passando para 80.

Nas décadas de 70 a 80 a Escola de Enfermagem de Ribeirão Preto - USP passa por transformação didático administrativas decorrentes de vários fatores:

- o parecer 163/72 que fixa as habilitações em enfermagem;

- o início da pós-graduação - nível mestrado e a especialização na área de Enfermagem Psiquiátrica em 1975;

- regulamentação da Comissão de Ensino como órgão assessor da direção da Escola, responsável pela coordenação didática.

- a transferência do curso de Auxiliar de Enfermagem para o Hospital das Clínicas;

- a ampliação de seu corpo docente.

O marco conceitual do ensino de enfermagem neste período se volta para a formação do enfermeiro para o mercado de trabalho. A participação da escola junto ao serviço de saúde da comunidade se amplia com a integração de seus docentes na Secretaria Estadual de Saúde, Prefeitura Municipal e Escolas de $1^{\circ}$ e $2^{\circ}$ graus.

$\mathrm{Na}$ década de 80 questões apontadas pela OMS/OPS, e as discussões no setor saúde vinculadas ao Plano Nacional de Saúde e as novas orientações do Ministério da Saúde e, da Previdência Social vêm reforçar a orientação do ensino de enfermagem voltado para as políticas de extensão de cobertura e de atenção primária em saúde.

Nesta década inicia-se o estudo da reforma curricular desta escola, tendo como marco referencial para o currículo:

- "o currículo de formação profissional mais do que um rol de disciplina é a seqüência de experiências através das quais a escola tenta estimular o desenvolvimento do aluno". Tais experiências devem ser planejadas, selecionadas e organizadas de modo a proporcionar o máximo de crescimento pessoal para o aprendiz, em todas as dimensões de sua vida intelectual, efetiva e motora, além de garantir-lhe integração social. (Comissão de Reestruturação Curricular da Escola de Enfermagem de Ribeirão Preto - USP, 1993).

A proposta de reformulação voltada para formação do enfermeiro generalista compreende o desenvolvimento do ensino centrado nas competências das diversas áreas de atuação profissional, compatível com experiências de aprendizagem nas quais o desempenho do aluno pressupõe fundamentação teórica para atuação nos serviços de saúde em diferentes níveis de complexidade, através de uma visão global, integrada e crítica da saúde. Esta reformulação generalista implica na competência técnico-científica e política a ser atingida ao longo do currículo de graduação. Tal competência decorre de uma reflexão acerca do homem, da sociedade, do processo saúde-doença, pressupondo uma abordagem multidisciplinar fundamentada 
nas ciências humanas, sociais e biológicas, e no saber próprio da enfermagem como profissão, com ênfase nos aspectos administrativos a serem desenvolvidos em instituições hospitalares e sanitárias.

O ciclo pré-profissional compreende os princípios gerais básicos de um currículo de formação profissional para a área da saúde, compondo o núcleo de ciências do comportamento, da biologia, da epidemiologia e da educação em saúde. O ciclo profissional compreende uma seqüência de experiências de aprendizagem dirigida à criança, à mulher ao adulto nas áreas de enfermagem clínica, cirúrgica, obstétrica, ginecológica, pediátrica e psiquiátrica. O último semestre do curso enfatiza experiência de aprendizagem voltada para a formação do enfermeiro generalista, capacitando-o para atuar na administração dos serviços básicos de saúde e da área hospitalar.

\section{O ENSINO DA ENFERMAGEM E SUAS PROSPECTIVAS}

Frente as atuais políticas de saúde e ao processo de municipalização neste setor cabe uma reflexão sobre alguns tópicos que favoreçam a compreensão da participação do enfermeiro e da escola enquanto órgão formado, neste processo ainda em organização:

- o papel das Universidades na reorganização dos serviços de saúde;

- a formação do enfermeiro e sua inserção no mercado de trabalho;

- a proposta de um currículo mínimo para a formação do enfermeiro.

\subsection{O papel da Universidade na reorganização dos serviços de saúde}

Cabe inicialmente, considerar como fica a universidade neste processo de reestruturação dos serviços de saúde frente a proposta do SUS, questionando-se a sua responsabilidade na formação de recursos humanos necessários à viabilização de tal projeto. Considerando que o SUS, aprovado pela Constituinte, é uma conquista inegável do povo brasileiro, e que os hospitais de ensino mantém a maior parte dos leitos hospitalares do país, a ANDES (Assessoria Nacional de Docentes do Ensino Superior) aponta para a necessidade de se recuperar a função de produção e transmissão de conhecimento destes estabelecimentos de ensino, diante do risco eminente de transformá-los em meras instituições assistencialistas. Algumas ações que visam a participação da Universidade neste projeto são apontadas:

- formação, capacitação e educação continuada de recursos humanos na área da saúde; 
- pesquisa científica e produção de tecnologia de interesse do SUS;

- transferência de tecnologia para o SUS;

- assistência de saúde a população;

- colaboração na formulação das políticas de saúde;

- assessoria as instâncias diretivas do SUS nos assuntos de competência das Instituições de Ensino Superior.

No momento em que se discute a questão da saúde, na esfera eminentemente política e de reorientação dos serviços de saúde a âmbito municipal inclusive, é importante que a enfermagem possa refletir sobre sua contribuição na formação e capacitação de seus exercentes, tanto no que diz respeito à formação do profissional de nível superior, como na capacitação do contingente de pessoal de nível médio e elementar.

Neste sentido deve-se pensar a formação do profissional voltada para uma prática onde se possibilite:

a) sistematização do processo administrativo desenvolvido pelo enfermeiro nos serviços de saúde;

b) a proposição de modelos assistenciais aos indivíduos e a coletividade;

c) desenvolvimento da pesquisa e da tecnologia na enfermagem;

d) a competência do profissional para a capacitação do pessoal de nível médio e elementar incluindo aqui a supervisão e controle da assistência prestada; participação do enfermeiro nas instâncias e formulação política e de assessoramento nas questões pertinentes a sua profissão;

e) a responsabilidade de resgatar os hospitais de ensino como centro de formação e desenvolvimento de novos conhecimentos e de novas propostas educacionais.

\subsection{A formação do enfermeiro e sua inserção no mercado de trabalho}

No que se refere a inserção do enfermeiro na forca de trabalho em saúde, este representa $2 \%$ desta força (de trabalho em saúde), sendo que as demais categorias auxiliares de nível médio e elementar correspondem a aproximadamente $70 \%$, onde os atendentes se apresentam em maior percentual. $\mathrm{Na}$ organização interna da força de trabalho em enfermagem, o enfermeiro participa com $8,5 \%$, os técnicos com $6,6 \%$, os auxiliares com $21 \%$ e os atendentes com $63 \%$, variando estas proporções nas diferentes regiões do país, porém mantendo as características.

Vale ressaltarmos as disparidades observadas na absorção do pessoal de enfermagem, na rede hospitalar privada e pública, na rede ambulatorial da rede básica de saúde como resultado do processo de municipalização. Guardando as proporções acima referidas, depreendemos que o enfermeiro está presente em maior número na rede pública, e os atendentes prevalecem na rede privada.

Neste sentido constatamos ainda a precariedade da situação empregatícia na 
enfermagem, as condições adversas de trabalho, a má remuneração, carga horária de trabalho excessiva, impropriedade dos instrumentos legais de proteção social e a discriminação no trabalho feminino.

Ao lado desta situação vivenciamos a polarização do trabalho em saúde, em seus dois extremos: o médico ditando normas para o cuidado ao paciente, através do seu poder de decisão sobre o processo terapêutico e o atendente executando o cuidado direto.

Quando apenas duas categorias integram quase 70\% do pessoal da saúde constatamos, uma situação anômala, que a própria organização dos serviços e atuação dos vários profissionais da saúde, tentam minimizar e corrigir, na medida em que as propostas de integralização e descentralização para o setor saúde, exigem uma composição multiprofissional desta equipe e a formação do enfermeiro voltada para atender as especificidades da organização deste setor.

O papel do enfermeiro tradicionalmente voltado a supervisão do pessoal auxiliar, à administração de unidades de internação centrada no modelo Taylorista, e ocasionalmente à prestação de cuidados diretos, impede-o muitas vezes de interferir efetivamente na assistência de enfermagem, mantendo-o distante das necessidades específicas de sua clientela, não Ihe permitindo uma visão global do setor saúde. (ALMEIDA, 1986).

A VIII Conferencia Nacional de Saúde, norteada dos princípios da Reforma Sanitária, leva-nos a questionar e rever as funções exercidas pelo enfermeiro. A proposta de um atendimento integral, no que se refere à promoção, proteção e recuperação da saúde, implica numa humanização do atendimento, a partir de uma visão global do homem na plenitude de seus direitos de cidadão. Nessa perspectiva entendemos que a formação do enfermeiro e demais categorias do setor enfermagem, deva estar vinculada as premissas básicas que estão norteando o movimento no setor saúde.

O corpo de conhecimento em enfermagem deve abranger uma formação que assegure ao profissional competência técnica, científica, administrativa e política, uma vez que de acordo com ALMEIDA (1986) "o objeto de trabalho do enfermeiro passa pela assistência da enfermagem no processo saúde-doença e se estende a organização do processo de trabalho em enfermagem". O currículo de formação profissional do enfermeiro, atualmente revisto em suas bases legais contempla necessariamente estas questões para a formação do profissional.

A competência técnico-científica leva o enfermeiro a atuar nos diferentes níveis de atenção a saúde (primário, secundário e terciário) e esta formação se respalda por um corpo de conhecimentos que contemple as questões relativas ao homem brasileiro em sua realidade concreta e histórica, bem como os aspectos bio-psico-sociais que o integram. A hierarquização da assistência a saúde demanda uma especialização para cada nível. Esta formação mais geral inclui conhecimentos fundamentados nas ciências biológica, na epidemiologia, estatística, nas ciências humanas e sociais e no processo tecnológico aplicado a saúde. 
A competência administrativa vem requerer o exercício das funções de coordenação, ensino e supervisão, a partir de novos modelos de administração. Esta formação passa a ser contemplada no currículo pelas disciplinas sobre planejamento, economia, informática, educação e outras, qualificando o profissional a exercer múltiplas funções no conjunto de ações que definem a assistência de enfermagem a ser prestada nos diferentes níveis de assistência saúde.

Por último, a competência política, desenvolvida a partir da competência técnica, cientifica e administrativa, exigirá do profissional o desenvolvimento de uma consciência mais crítica, refletindo-se em uma ação participativa e transformadora na prática da saúde.

A esse respeito, ALMEIDA (1986) enfatiza alguns princípios que passamos a considerar:

- que a saúde não é exclusivamente possibilitar acesso aos serviços de saúde, mas é um processo mais amplo que inclui condições de vida, como trabalho, moradia, transporte, educação, renda, lazer;

- que a função do enfermeiro não é só a assistência, mas tem um componente de ação transformadora da realidade em que atua;

- que o ensino da enfermagem deve se preocupar com a garantia da integração docenteassistencial, ampliando os campos de estágios para além dos hospitais de ensino tradicional e unidades básicas, com presença nos órgãos de decisão das políticas de saúde, tanto a nível local, quanto regional;

- que na formação do enfermeiro as questões relativas a condição de trabalho sejam de acordo com os princípios contidos na VIII Conferência Nacional de Saúde.

\subsection{A proposta de um novo currículo mínimo para a formação do enfermeiro}

O documento sobre a Proposta Preliminar de Currículo Mínimo para o curso de Graduação em Enfermagem, em tramitação junto ao Conselho Federal de Educação vem de certa forma, consolidar algumas diretrizes apontadas pela VIII Conferência Nacional de Saúde, e a Conferência de Recursos Humanos em Saúde. Este documento define que cabe aos enfermeiros a competência para desenvolverem atividades em quatro áreas fundamentais - de - assistência, gerência, ensino e produção científica.

Tal proposta reforça fundamentalmente a formação do enfermeiro para o processo de trabalho em enfermagem, a partir dos princípios de uma educação transformadora que incite a reflexão e indagação sobre a prática profissional e a valorização do compromisso do enfermeiro para a com a sociedade.

A proposta de novo currículo tem como referencial uma visão crítica das condições de vida e o perfil epidemiológico da população, das diretrizes políticas definidas para o setor saúde, da demanda dos serviços de saúde, e a função e o espaço profissional neste contexto. 
Baseados nestes princípios, o documento define o perfil do profissional a partir de uma formação generalista, levando ao desenvolvimento de competências técnico-político nas áreas assistencial, administrativa, pedagógica e de pesquisa, para cuidar do ser humano em todo seu ciclo evolutivo, ao nível da promoção e recuperação da saúde. (ASSOCIAÇÃO BRASILEIRA DE ENFERMAGEM, 1989).

Esta proposta visa consolidar o ensino da enfermagem, não mais sob um modelo tradicionalista, mas sim pautado em um novo modelo educacional voltado para a formação de um profissional mais crítico e participativo, atuando nos diferentes níveis de assistência. Caminha, sobretudo para uma postura emancipatória, que, tem como princípios a crítica e a ação. (SAUL, 1989).

$\mathrm{Na}$ análise de SAUL (1989), rever o currículo na perspectiva de um modelo de racionalidade emancipatória implica em situar a escola, como aparelho formador, no contexto social. Implica, sobretudo, em estabelecer uma relação dialética entre conteúdos, método, contexto histórico, social e cultural no processo de formação profissional. O currículo baseado neste paradigma tem como meta de educação a formação de um cidadão trabalhador.

Objetivando a formação do enfermeiro, isto significa dizer que sua formação técnicocientífica deve se fazer rigorosa, passando necessariamente pela reflexão crítica das questões sociais, onde o profissional atua. A capacitação do pessoal docente, do ponto de vista pedagógico e filosófico deverá estar objetivamente vinculado a um conceito educacional. Isto exigirá a necessidade de programas de educação continuada para os próprios docentes, visando prepará-los para os desafios que surgem no campo do ensino e da capacitação profissional, diante de uma nova perspectiva educacional que necessariamente levará a mudanças concretas no ensino e na prática profissional.

\section{CONCLUSÃO}

Para se estruturar um novo modelo educacional para a enfermagem, necessário se faz que as Escolas de Enfermagem se transformem, no sentido de garantir a formação do profissional com competência e adequada base técnica-científica e política. Esta formação se dará nos diferentes níveis de serviços de assistência à saúde, sendo a reorganização destes serviços e a constituição da rede básica, elementos centrais desta estratégia de integração do ensino com a prática profissional. As escolas poderão, a partir daí, orientar seus currículos de maneira coerente com estes princípios, considerando o conjunto dos serviços de saúde e não apenas o hospital de ensino. A estrutura curricular deve ser orientada no sentido de uma maior integração das disciplinas do ciclo pré-profissional, que irão fundamentar 
o conhecimento técnico-científico e das disciplinas do ciclo profissional objetivando consolidar a assistência prestada nos diferentes níveis de complexidade, superando o enfoque do currículo vigente, centrado nas especializações médicas. A capacitação do pessoal docente, do ponto de vista pedagógico e filosófico está objetivamente vinculada a um novo conceito educacional, o de que a formação é resultado da relação didática entre a prática e a teoria, que começa e termina com a primeira.

\section{THE UNDERGRADUATE EDUCATION AT THE COLLEGE OF NURSING, UNIVERSITY OF SÃO PAULO AT RIBEIRÃO PRETO CAMPUS - ITS PROSPECTIVES}

Authors focused the undergraduate education at the College of Nursing, University of São Paulo at Ribeirão Preto Campus, through its historical and conceptual mark since its establishment in the 1950's. In the 1980's, a study of curriculum modification originated from a wide process of discussion, culminated in a proposal of formation of generalized nurses and the introduction of a new curriculum in 1989.

According to authors' version, the prospectives to nursing education depend on the schools' reflection about the University's role in the reorientation of health services and formation of students aiming at political, scientific and technical competence to actuate at different levels of community health assistance. A new educational model will require a curriculum reorientation and the insertion of schools in the health services as a strategic component of the integration of education with professional practice.

UNITERMS: nursing education, under graduation teaching.

\section{LA ENSEÑANZA DE GRADUACIÓN LA ESCUELA DE ENFERMERÍA DE RIBEIRÃO PRETO DE LA UNIVERSIDAD DE SÃO PAULO - SUS PROSPECTIVAS}

Las autoras abordan la enseñanza de graduación de la Escuela de Enfermería de Ribeirão Preto - USP, a través de su marco histórico y conceptual desde su fundación en la década del 50. En la década del 80, el estudio de la reforma curricular derivó de un amplio proceso de discusión, culminando con la propuesta de formación del enfermero generalista y la implantación del nuevo curriculum en 1989. 
En la versión de las autoras, las prospectivas para la enseñanza de la graduación dependen de una reflexión de las escuelas sobre el papel de las Universidades en la reorientación de los servicios de salud, de la formación del alumno orientada para la competencia técnica, científica, política para actuar en diferentes niveles de asistencia a la salud de la población. Un nuevo modelo educacional exigirá la reorientación de los curriculums y la inserción de las escuelas en los servicios de salud como elementos estratégico de integración de la enseñanza con la práctica profesional.

UNITERMOS: enseñanza de pre-grado, educación en la enfermería

\section{REFERÊNCIAS BIBLIOGRÁFICAS}

01. ALMEIDA, M. C. P. et al. A formação do enfermeiro frente a reforma sanitária. Cadernos de Saúde Pública. Rio de Janeiro, v. 2, n. 4, p. 505-10, out/dez, 1986.

02. ASSOCIAÇÃO BRASILEIRA DE ENFERMAGEM. COMISSÃO DE EDUCAÇÃO. Proposta preliminar de currículo mínimo para os cursos de graduação em enfermagem. Brasília: SESU/MEC; Comissão de especialistas em enfermagem; Comissão de Educação da ABEn; mar., 1989 (Mimeografado).

03. COMISSÃO DE REESTRUTURAÇÃO CURRICULAR DA ESCOLA DE ENFERMAGEM DE RIBEIRÃO PRETO-USP. Reforma curricular de graduação em enfermagem. Escola de Enfermagem de Ribeirão Preto. Universidade de São Paulo. Ver. LatinoAmericana de Enfermagem. Ribeirão Preto, v. 2, n. 1, p. 35-52, jul., 1993.

04. SAUL, A. M. A formação como determinante da competência profissional. In: ASSOCIAÇÃO BRASILEIRA DE ENFERMAGEM. Perfil e competência do enfermeiro e o currículo mínimo de graduação em enfermagem. Seminário da Região Sudeste, nov., 1988. Anais. São Paulo: Associação Brasileira de Enfermagem, 1988. p. 106. 